\title{
Wavelet Analysis to Detect Multi-scale Coherent Eddy Structures and Intermittency in Turbulent Boundary Layer
}

\author{
Jiang $\operatorname{Nan}^{1,2}$ \\ ${ }^{1}$ Department of Mechanics, Tianjin University \\ 2Tianjin Key Laboratory of Modern Engineering Mechanics,
}

China

\section{Introduction}

In the early stage of turbulence study, turbulent flow was deemed fully random and disorder motions of fluid particles. Thus physical quantity describing turbulence was considered as the composition of random fluctuations in spatial and temporal field. Reynolds (1895) divided the turbulent field into mean field and fluctuating field and then theories and methods based on statistics for turbulence research were developed. Kolmogorov ${ }^{[1]}$ analyzed the relative motion of fluid particles in fully development isotropic and homogeneous turbulent flow based on random field theory and presented the concept of structure functions, which described the relative velocity of two fluid particles separated by distance of 1 , to investigate the statistical scaling law of turbulence:

$$
<|\delta \mathrm{u}(\mathrm{l})|^{\mathrm{p}}>\propto 1^{\zeta(\mathrm{p})} \quad \eta<<\mathrm{l}<<\mathrm{L}
$$

Where $\delta \mathrm{u}(\mathrm{l})=\mathrm{u}(\mathrm{x}+\mathrm{l})-\mathrm{u}(\mathrm{x})$ is the velocity component increment along the longitudinal direction at two positions $\mathrm{x}$ and $\mathrm{x}+1$ respectively separated by a relative separation $1, \eta$ is the Kolmogorov dissipation scale of turbulence, $\mathrm{L}$ is the integral scale of turbulence, $<>$ denotes ensemble average and $\zeta(\mathrm{p})$ is scaling exponent.

Kolmogorov (1941) ${ }^{[1]}$ successfully predicted the existence of the inertial-range and the famous the linear scaling law which is equivalent to the $-5 / 3$ power spectrum:

$$
\zeta(\mathrm{p})=\frac{\mathrm{p}}{3}
$$

Because of the existence of intermittence of turbulence, scaling exponents increases with order nonlinearly which is called anomalous scaling law. In 1962, Kolmogorov[2] presented Refined Similarity Hypothesis, and thought that the coarse-grained velocity fluctuation and the coarse-grained energy dissipation rate are related through dimensional relationship:

$$
<\delta \mathrm{u}(\mathrm{l})>\propto\left(\varepsilon_{1}\right)^{1 / 3}
$$




$$
<\varepsilon_{1}^{\mathrm{p}}>\propto 1^{\tau(\mathrm{p})}
$$

Where $\varepsilon_{1}$ is the coarse-grained turbulent kinetic energy dissipation rate over a ball of size of 1 .

So it yields the relationship between the scaling exponent $\zeta(\mathrm{p})$ for the velocity structure function and the scaling exponent $\tau_{\mathrm{p}}$ for the turbulent kinetic energy dissipation rate function as:

$$
\zeta(\mathrm{p})=\frac{\mathrm{p}}{3}+\tau\left(\frac{\mathrm{p}}{3}\right)
$$

Jiang ${ }^{[3]}$ has demonstrated that scaling exponents of turbulent kinetic energy dissipation rate structure function is independent of the vertical positions normal to the wall in turbulent boundary layer, so the scaling law of dissipation rate structure function is universal even in inhomogeneous and non-isotropic turbulence. However, scaling exponent, $\zeta(\mathrm{p})$, is very sensitive to the intermittent structures and is easy to change with the different type of shear flow field because the most intermittent structures change with spatial position and direction[4]-[8]. The systematic change of $\zeta(\mathrm{p})$ shows the variation of physical flow field [9]. Scaling exponent, $\zeta(\mathrm{p})$, has been found to be smaller in wall turbulence than that in isotropic and homogeneous turbulence by G Ruiz Chavarria[5] and F.Toschi[6][7] both in numerical and physical investigations. The scaling laws appear to be strongly depending on the distance from the wall. The increase of intermittence near the wall is related to the increase of mean shear of velocity gradient.

After 1950s', turbulent fluctuation was extendedly studied with the development of experimental technique of fluid mechanics. Large-scale motions, which were relatively organized and intermittent, were found in jet flow, wake flow, mixing layer and turbulent boundary layer. This kind of large-scale structure was universal and repeatable on intensity, scale shape and process to a certain type of shear flow. So it was called coherent structure (or organized motion). Research on coherent structure done by Kline group (1967) [10] of Stanford University, a great breakthrough in the study of turbulent boundary layer, found the low-speed streak structure and burst in the near wall region. This result, which has been verified by Corino(1969) [11] Kim (1971) ${ }^{[12]}$ and Smith (1983)[13], is one of a few conclusions universally accepted in this field. The discovery of coherent structures, a great breakthrough in turbulent study, which has greatly changed traditional view of turbulent essence, indicates the milestone of study on turbulence essence from disorder stage to organized stage ${ }^{[14]}$.

Coherent structures exist not only in large scales, but also in small scales [15][16]. Indeed, as indicated by Sandborn [17] in 1959, who analyzed band passed signals, the presence of low speed streaks might be indicated by "bursts in the over all frequencies". In recent years, universal and organized small-scale coherent structures have been discovered in turbulent flows. The recently experimental measurements and DNS results present that small-scale filamentary coherent structures also exist in homogeneous and isotropic turbulence [18]-[21]. G Ruiz Chavarria [5], F.Toschi[6][7], Ciguel Onorato[8] R. Camussi[15], T. Miyauchi[16] discovered that small-scale coherent structures also exist in turbulent channel flow and turbulent boundary layer with strong intermittency. Using the detection criterion for multi-scale coherent eddy structure, the anomalous scaling law, as well as intermittency of turbulence, 
is found to be dependent on the probability density functions of structure function characterized by increasingly wider tails [8][22].

However, in spite of all of above improvements, the dynamical mechanism and behavior of multi-scale coherent structure has been unclear. The relationship between the statistical intermittency and the dynamics for the multi-scale coherent structure still remains poorly understood. Researchers are very actively trying to explain the underlying physical mechanism of intermittency and multi-scale coherent structures in shear turbulence. Dynamical description of intermittency and multi-scale coherent structures in shear turbulence has become one of the most fascinating issues in turbulence research. The advance of research on the intermittency of multi-scale coherent structures in shear turbulence have an important impact on establishing more effective numerical simulation method and sub-grid scale model based on the decomposition of multi-scale structures. Characterizing the intermittency of multi-scale coherent structures in shear turbulence in terms of their physics and behavior still should be undertaken as a topic of considerable study. Farge [23] has recently presented a coherent vortex simulation method instead of wave number decompositions generally used. This new method is in coincidence with the physical characteristics of turbulence and provides a new access to direct numerical simulation. Charles Meneveau [24] has recently advanced some new physical concepts, such as turbulent fluctuation kinetic energy, transfer of turbulent fluctuation kinetic energy, flux of turbulent fluctuation kinetic energy and so on, which is the foundation to set up more effective turbulence model and sub-grid scale model.

In this chapter, we concentrate on some fundamental characteristics of intermittency and multi-scale coherent structures in turbulent boundary layer. We separate turbulence fluctuating velocity signals into two components based on information of wavelet transform, one component containing multi-scale coherent structure characterized by intermittency, while the other containing the remaining portion of the signal essentially characterized by the random component. The organization is as follow: in section 2, wavelet transform and its applications to turbulence research is introduced. In section 3, the experimental apparatus and technique are described. The results and discussion are given in section 4 and finally, conclusions are drawn in section 5 .

\section{Multi-scale coherent eddy structure detection by wavelet transforms}

\subsection{Wavelet transform}

Wavelet transform ${ }^{[25]}$ is a mathematic technique developed in last century for signals processing. It convolutes signals with an analytic function named wavelet at a definite position and a definite scale by means of dilations and translations of mother wavelet. It provides a two-dimensional unfolding of one-dimensional signals resolving both the position and the scale as independent variables. So it comprises a decomposition of signals both on position and scale space simultaneously.

Wavelet is a local oscillation or perturbation with definite scale and limited scope in certain location of physical time or space. If a function $\psi(\mathrm{t}) \in \mathrm{L}^{2}(\mathrm{R})$ satisfies the socalled "admissibility" condition :

$$
\mathrm{C}_{\psi}=\int_{-\infty}^{+\infty} \frac{|\hat{\psi}(\omega)|^{2}}{|\omega|} \mathrm{d} \omega<+\infty
$$


Where $\hat{\psi}(\omega)$ is the Fourier transform of $\psi(\mathrm{t}), \psi(\mathrm{t})$ is called a "mother wavelet".

Relative to every mother wavelet $\psi(\mathrm{t}), \psi_{\mathrm{ab}}(\mathrm{t})$ is the translation (by factor $\mathrm{b}$ ) and dilatation (by factor $\mathrm{a}>0$ ) of $\psi(\mathrm{t})$ :

$$
\psi_{\mathrm{ab}}(\mathrm{t})=\frac{1}{\sqrt{\mathrm{a}}} \psi\left(\frac{\mathrm{t}-\mathrm{b}}{\mathrm{a}}\right) \text { with } a, b \in R \text { and } \mathrm{a}>0
$$

The wavelet transform $\psi_{\mathrm{f}}(\mathrm{a}, \mathrm{b})$ of signal $\mathrm{s}(\mathrm{t}) \in \mathrm{L}^{2}(\mathrm{R})$ with respect to $\psi_{\mathrm{ab}}(\mathrm{t})$ is defined as their scalar product defined by:

$$
\psi_{\mathrm{s}}(\mathrm{a}, \mathrm{b})=\int_{-\infty}^{+\infty} \mathrm{s}(\mathrm{t}) \overline{\psi_{\mathrm{ab}}(\mathrm{t})} \mathrm{dt}
$$

The total energy of the signal can be decomposed by :

$$
\mathrm{E}=\int_{-\infty}^{+\infty}|\mathrm{s}(\mathrm{t})|^{2} \mathrm{dt}=\int_{0}^{+\infty} \frac{\mathrm{da}}{\mathrm{a}^{2}} \int_{-\infty}^{+\infty} \frac{2}{\mathrm{C}_{\psi}}\left|\psi_{\mathrm{s}}(\mathrm{a}, \mathrm{b})\right|^{2} \mathrm{db}=\int_{0}^{+\infty} \frac{\mathrm{da}}{\mathrm{a}^{2}} \int_{-\infty}^{+\infty} \mathrm{I}(\mathrm{a}, \mathrm{b}) \mathrm{db}
$$

with

$$
\mathrm{I}(\mathrm{a}, \mathrm{b})=\frac{2}{\mathrm{C}_{\psi}}\left|\psi_{\mathrm{s}}(\mathrm{a}, \mathrm{b})\right|^{2}
$$

and

$$
\mathrm{E}(\mathrm{a})=<\mathrm{I}(\mathrm{a}, \mathrm{b})>_{\mathrm{b}}
$$

where \langle\rangle$_{b}$ denotes ensemble average over parameter $b$.

Equation (5) is the local wavelet spectrum function and equation (6) is the multi-scale wavelet spectrum function respectively. Based on equation (5), the kinetic energy of signal is decomposed into one-to-one local structures with definite scale a at definite location $b$. Wavelet spectrum function defined by (6) means the integral kinetic energy on all structures with individual length scale a .

On the concept of wavelet transformation, skew factor of multi-scale eddy structure can be defined by wavelet coefficient as:

$$
\operatorname{Sk}(\mathrm{a})=\frac{\left\langle\psi_{\mathrm{s}}(\mathrm{a}, \mathrm{b})^{3}\right\rangle_{\mathrm{b}}}{[\mathrm{E}(\mathrm{a})]^{3 / 2}}
$$

Skew factor is the enhancement of wavelet coefficient $\psi_{\mathrm{f}}(\mathrm{a}, \mathrm{b})$, which is capable of revealing the signal variation across scale parameters. So skew factor is the qualitative indicator of intermittency of multi-scale structure.

Another indicator of intermittency is the flatness factor of the wavelet coefficients:

$$
F F(a)=\frac{\left\langle\psi_{s}(a, b)^{4}\right\rangle_{b}}{[E(a)]^{2}}
$$


Flatness factor is the enhancement of the amplitude of wavelet coefficient $\psi_{\mathrm{f}}(\mathrm{a}, \mathrm{b})$ in spite of its sign, which is capable of revealing the amplitude difference of wavelet coefficient across scale parameters.

\subsection{Wavelet and turbulence eddy}

Wavelet transform provides the most suitable elementary representation of turbulent flows. "Eddies" are the fundamental element in turbulent flows. As TENNEKES \& LUMLEY[27] pointed out "An eddy, however, is associated with many Fourier coefficients and the phase relations among them. Fourier transforms are used because they are convenient (spectra can be measured easily); more sophisticated transforms are needed if one wants to decompose a velocity field into eddies instead of waves." Eddy and wavelet share common features in many physical aspects, and wavelet can be regarded as the mathematical mode of an eddy structure in turbulent flows[28][29]. As a new tool, wavelet transform can be devoted to identify coherent structure in wall turbulence instead of the conditional sampling methods traditionally used. JIANG[30] has performed the wavelet decompositions of the longitudinal velocity fluctuation in a turbulent boundary layer. The energy maximum criterion is established to determine the scale that corresponds to coherent structure. The coherent structure velocity is extracted from the turbulent fluctuating velocity by wavelet inverse transform.

Figure 1 presents the time trace signal of instantaneous longitudinal velocity measured by hot-wire probe in the buffer sub-layer of turbulent boundary layer with its wavelet coefficients contour transformed by wavelet transform. From the standard $(a, t)$ plane representation of the wavelet coefficients, it can be seen that there exist one-to-one events at different positions and different scales correspond to the signal. The large-scale eddies seem to be randomly distributed and are fairly space filling. A typical process in which a large eddy creates two or more small eddies can be seen clearly. This subdivision repeats until eddies reach the scale at which they are readily dissipated by the fluid viscosity. There is a kinetic energy flux from larger eddies to smaller ones. The smaller eddies obtain their energy at the expense of the energy loss in larger eddies. In turbulent boundary layer, the colorful spots have special physical meaning related to the coherent structures burst events which are the most important structures in wall turbulence and contribute most to the turbulence production in the near wall region. The red spots represent the accelerating events at different scales which are the high-speed fluids sweep to the probe and cause the high-speed velocity output from the hot-wire probe while the blue spots stand for the decelerating events which is the low-speed fluids eject from the near wall region to the probe and cause the low-speed velocity output from the hot-wire probe.

Figure 2(a) shows the typical shape of an "eddy" correlation function and spectral function proposed by TENNEKES \& LUMLEY[27] based on turbulence interpretation. Figure 2(b) shows the typical shape of a wavelet both in correlation function and spectral space. Figure 2(c) shows the shape of an "eddy" of turbulence both in correlation function and spectral space obtained by wavelet decomposition from turbulent flow in experimental measurement. From figure 2(a), figure 2(b) and figure 2(c), it can be found that they are fit each other. 

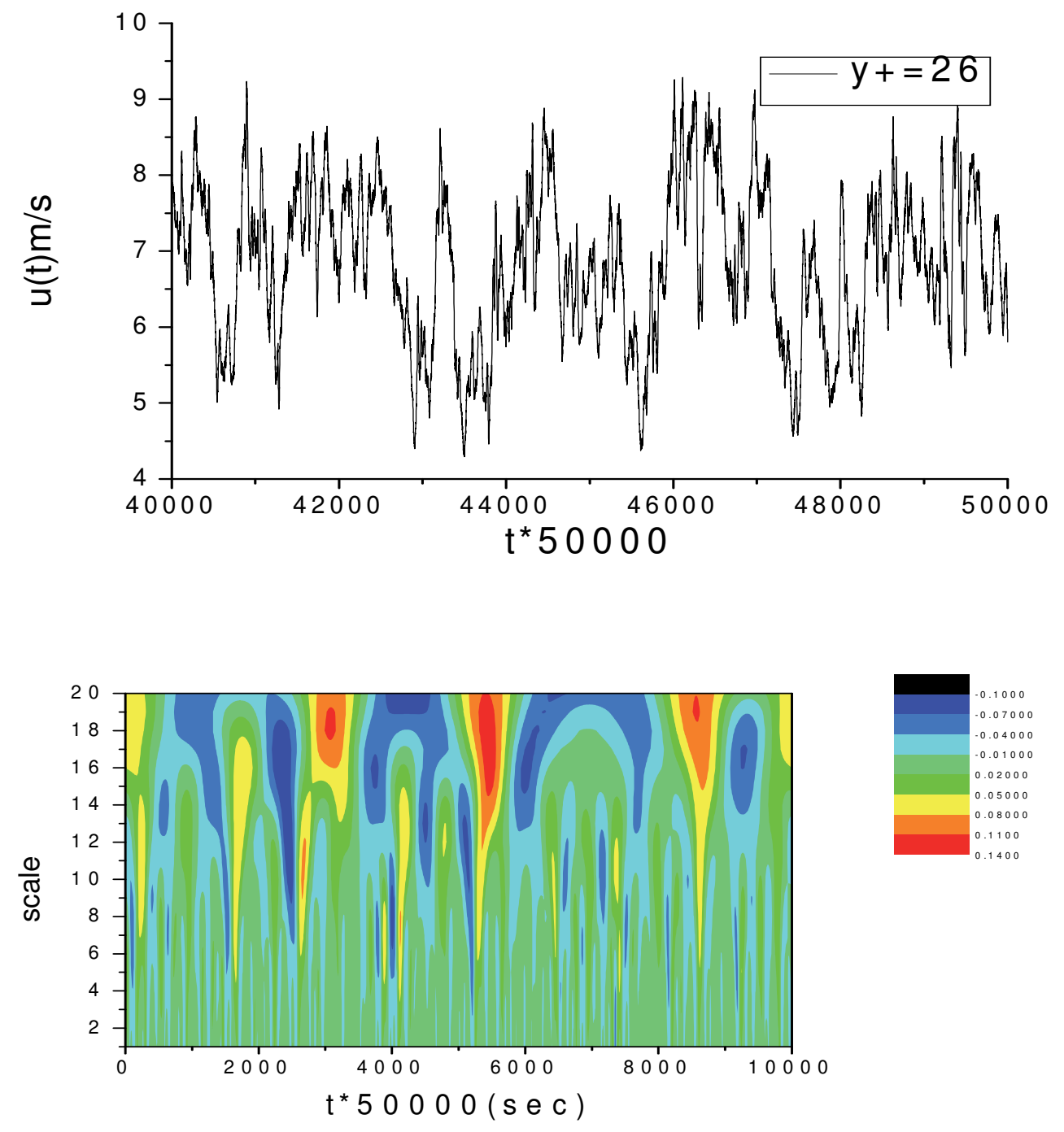

Fig. 1. Wavelet coefficients magnitude contour of the longitudinal fluctuating velocity signal 

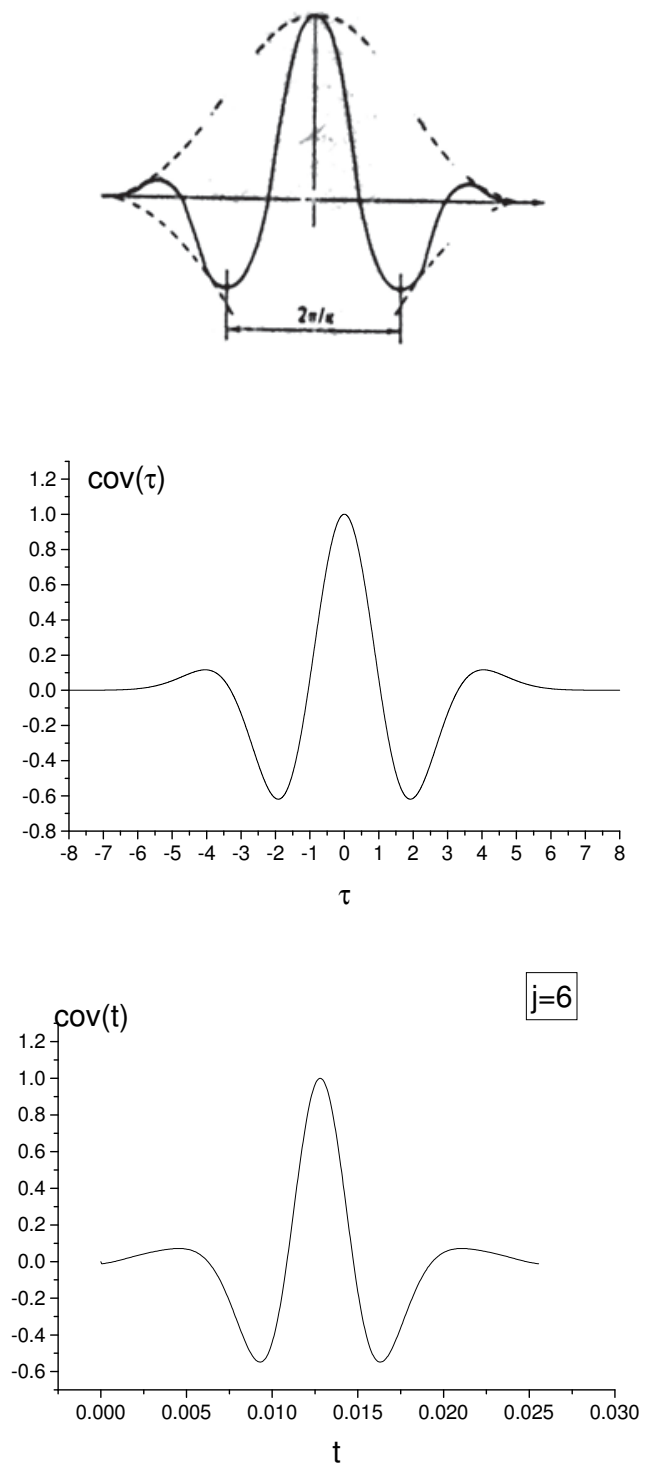

Fig. 2. An eddy typical shape defined by (a) TENNEKES \& LUMLEY[27] based on turbulence interpretation (b) a wavelet function(c) wavelet transform of turbulent flow

Figure 3 is the eddy structure velocity signals for each single scale decomposed by wavelet transform. Figure 4 is the correlation functions of them. They are in agreement with the concept of a typical "eddy" structure proposed by TENNEKES \& LUMLEY[27] for turbulence interpretation. The eddy wavelength for each scale can be measured between the troughs of the correlation functions as defined by TENNEKES \& LUMLEY[27] in figure 2(a). 

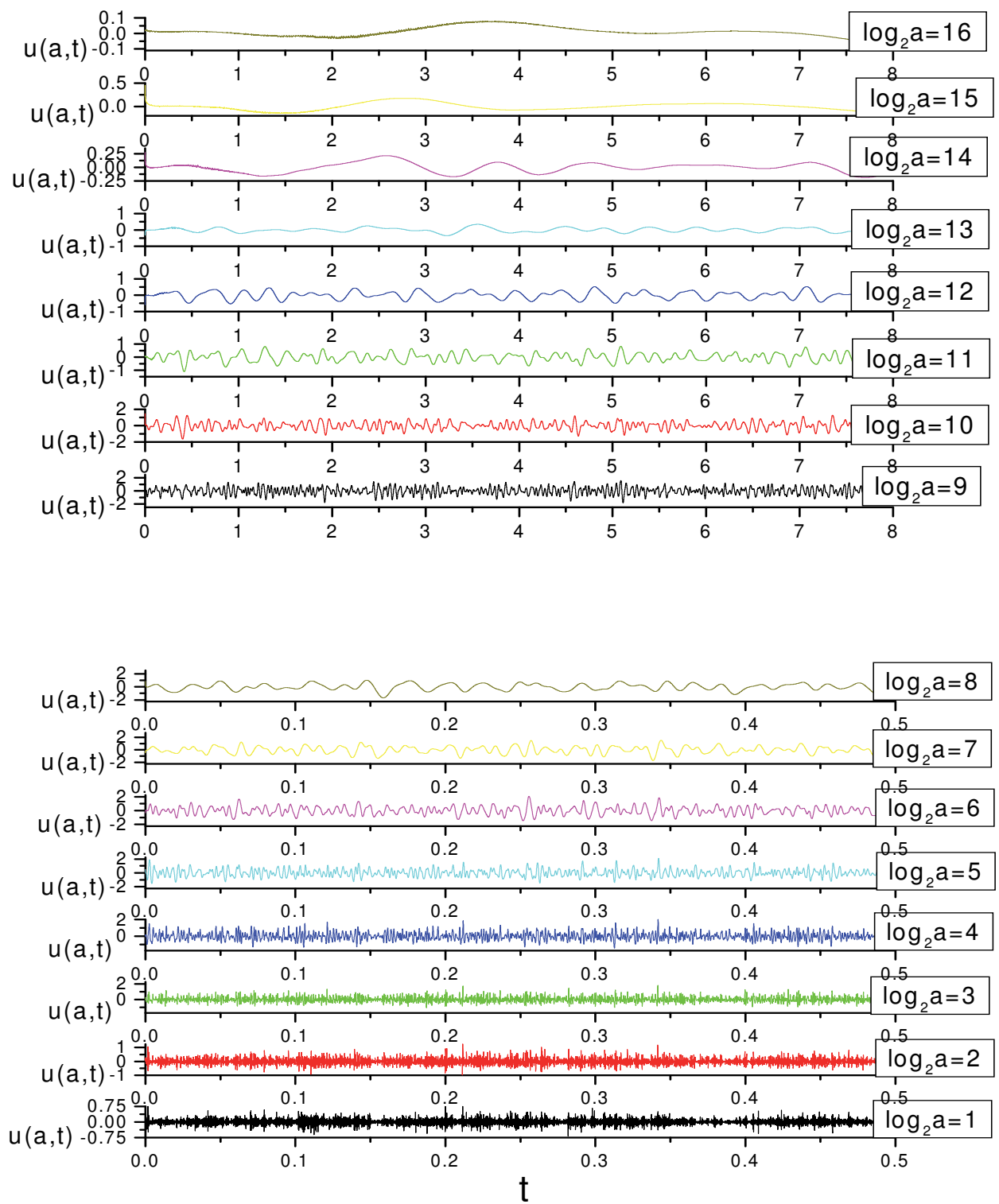

Fig. 3. Multi-scale eddy structure velocity decomposed by wavelet transformation of turbulence fluctuation 


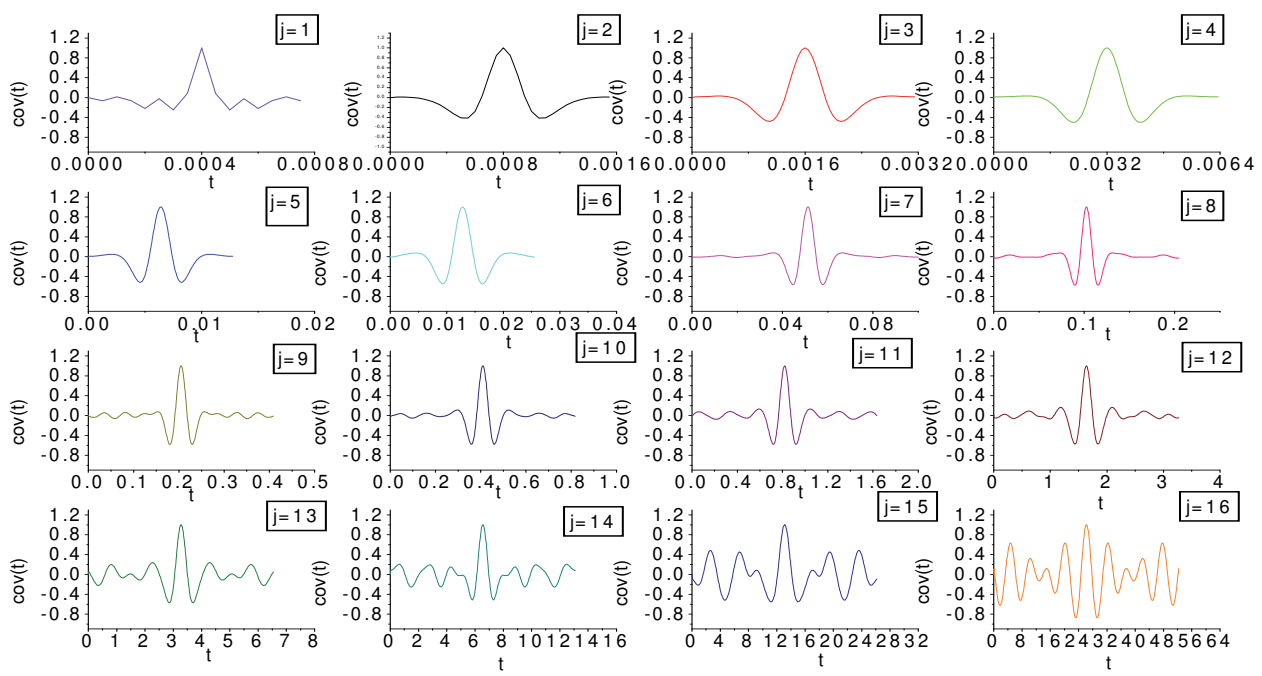

Fig. 4. Correlation functions and scale measurements of multi-scale eddy structures

In order to detect the multi-scale coherent eddy structure in turbulence, a educe method by conditional sampling scheme using the intermittency factor of wavelet coefficients, is used to extract the phase-averaged evolution course for multi-scale coherent eddy structures in wall turbulence. The method can be simply summarized as follows: computing the flatness factor $F F(a)$ at each wavelet scale, if $F F(a)$ is less then 3, coherent eddy structures are not detected and turn to the next scale. If $F F(a)$ is greater than 3 for a given scale, imposing a threshold level $L$ on $I(a, t)$ and excluding those wavelet coefficients whose $I(a, t)$ is greater than $L$, then recalculated the flatness factor $F F(a)$. If $F F(a)$ is less then 3, then turns to the next scale. If the flatness factor $F F(a)$ is still larger than 3, the threshold level $\mathrm{L}$ is lowered and the process is iterated until the flatness factor $F F(a)$ equals to (or less than) 3 for all scales[31][32].

Figure 5 shows the energy contribution of each scale eddies versus scale $a$ by integrating the square of the modulus of wavelet coefficients over the temporal location parameter $t$. It can be found from figure 5 that the energy distribution of each scale eddies are not constant and varies across scale parameter $a$. There is a scale that corresponds to the peak of energy contributions. This energy maximum is related to the large-scale coherent structures in the near region of turbulent boundary layer and is called burst. Coherent structures are found to be particularly important "eddies" and they are a major contribution to the production of turbulence in turbulent boundary layer. As can be seen, for buffer layer, the maximum energy scale is scale 10, while for logarithm-law layer; scale 9 is the maximum energy scale. The flatness factor $F F(a)$ calculated by averaging the 4-th power of the modulus of wavelet coefficients over the temporal location parameter $t$ at each scale $a$ is shown in Figure 6 . Flatness factor decreases with scale from significantly larger than 3 to about 3 . In comparison to Figure 5, the flatness factor at scales less than the most energetic scale correspond to the peak of energy contributions satisfies $F F(a)>3$, which indicates that lots of intermittent structures satisfying $\mathrm{FF}(\mathrm{a}, \mathrm{t})>3$, namely coherent structures, exist. While in 
scales larger than the most energetic scale corresponds to the peak of energy contributions, the flatness factors almost satisfy $F F(a)<3$, which indicates that few coherent structures satisfying $F F(a, t)>3$ exist.
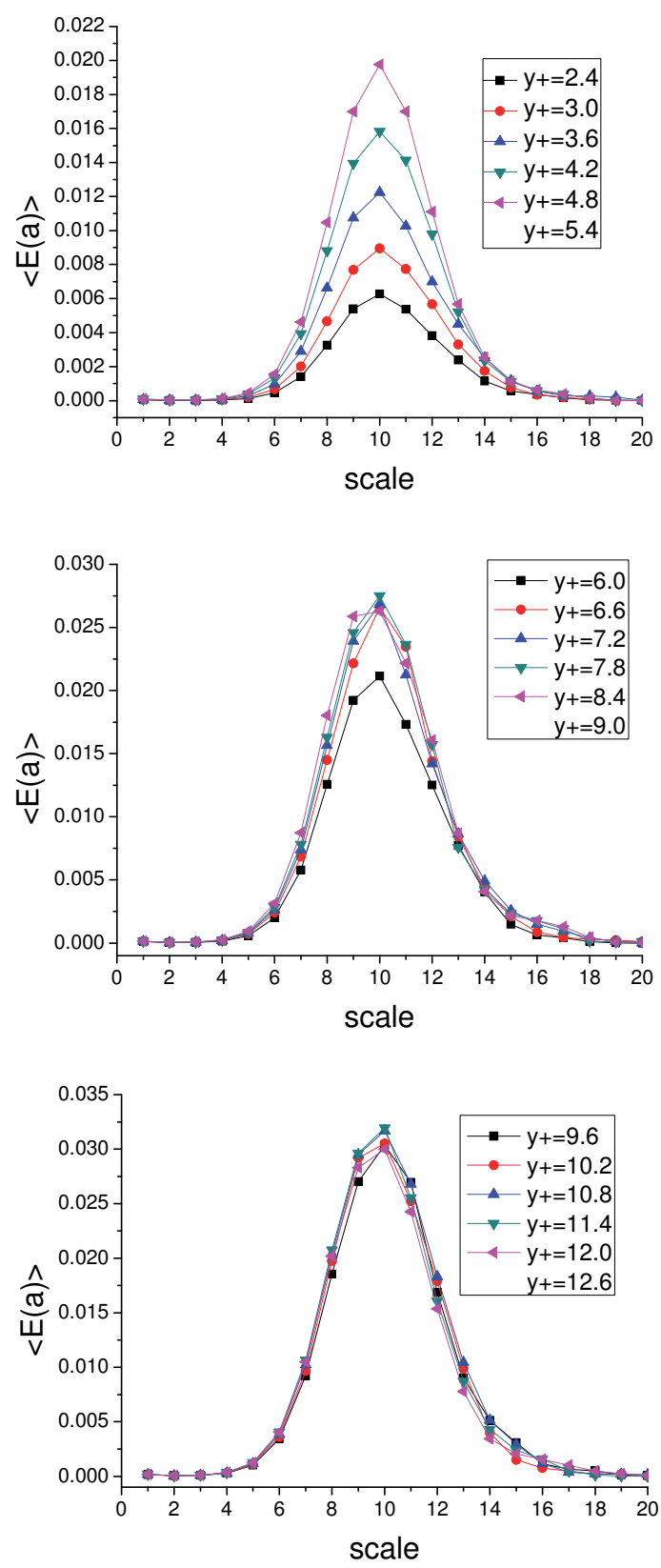

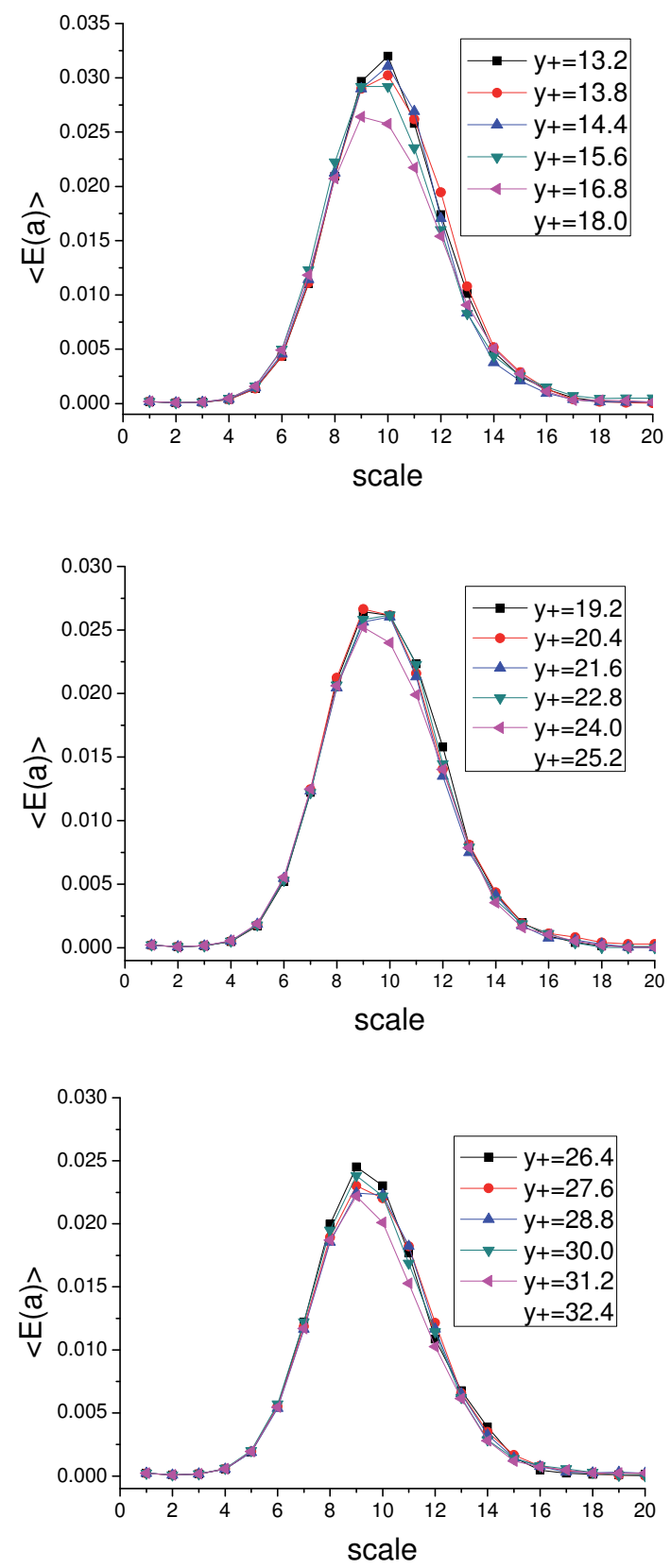

Fig. 5. Energy distribution as a function of wavelet scales at different locations in turbulent boundary layer 


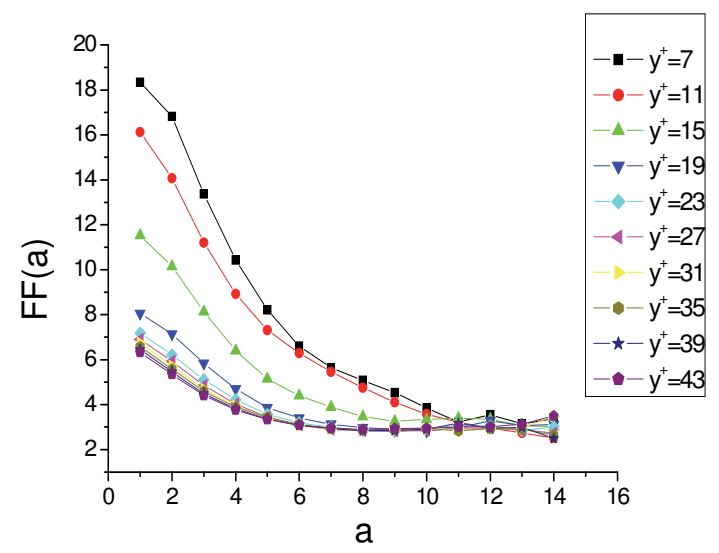

Fig. 6. Flatness as a function of wavelet scales at different locations in turbulent boundary layer

\section{Experimental apparatus and technique}

The experiment has been performed in a low turbulent level wind tunnel. The working section, length is $4500 \mathrm{~mm}$, with cross-section is welding rectangular of height $450 \mathrm{~mm}$ and width $350 \mathrm{~mm}$, adopted controllable silicon timing system, power of $7.5 \mathrm{Kw}$. Wind velocity in test section continuously varies from $1.0 \mathrm{~m} / \mathrm{s}$ to $50.0 \mathrm{~m} / \mathrm{s}$, and primal turbulent level is less than $0.07 \%$. The test flat plate is fixed on the horizontal center of the test section, parallel with the direction in which the wind comes. The leading edge of the plate faced to the wind direction is symmetry wedged. The length of plate is $4500 \mathrm{~mm}$, with width of $350 \mathrm{~mm}$ and thickness of $5 \mathrm{~mm}$. A piece of sandpaper stuck on the leading edge of the plate as a trigger to trip transition from laminar flow to turbulent flow and forms fully developed turbulence downstream. When the longitudinal velocity component in $10 \mathrm{~m} / \mathrm{s}$, the thickness of boundary layer is $\delta=160 \mathrm{~mm}$, Reynolds number $\operatorname{Re}_{\delta}=\frac{U_{\infty} \delta}{v}=100000$. IFA300 constanttemperature anemometer, made by TSI Corporation in America, is used to acquire the digital velocity signal output from the hot-wire probe, controlled by computer and has the best automatic frequency to deserve the best frequent correspondence instantaneously. The probe used in the experiment is TSI-1211-T1.5 hot-wire probe with single sensor. The temperature sensitive material is tungsten filament with diameter of $2.5 \mu \mathrm{m}$. The time sequence of longitudinal velocity component at 100 locations, with the nearest distance from the plate surface is $y=0.5 \mathrm{~mm}$ has been finely measured by IFA300 with resolution higher than Kolmogorov dissipation scale. For each measurement position, the sampling frequency is $50 \mathrm{~K}$, sampling time is $21 \mathrm{~s}, 1048576$ samples of the anemometer output signal are digitized in each database file by the 12-bit A/D converter of model UEI-WIN30DS4.Before measurement, each probe should be calibrated solely, in order to obtain the finest frequency correspondence and the relationship between output voltage and flow velocity. TSI-1128A type hot wire velocity calibrator can provide standard jet flow field with continuous velocity between 0 and $50 \mathrm{~m} / \mathrm{s}$ to calibrate the probe. The diameter of jet nozzle is $\mathrm{D}=10 \mathrm{~mm}$. The semi logarithmic mean velocity profile normalized by wall unit is given in Fig 7, where 
$u^{+}=\mathrm{U} / \mathrm{u}_{*}, y^{+}=\mathrm{yu}_{*} / v$. The skin friction velocity estimated by regression between $y^{+}=40$ and $y^{+}=200$ is $u_{\tau}=0.3906 \mathrm{~m} / \mathrm{s}$ and the skin friction coefficient is $c_{f}=0.0039$. Buffer layer, log-layer and bulk region can be distinguished in the single wall distance regions by their characteristic curvatures, while the linear viscous sub-layer region could not been resolved sufficiently.

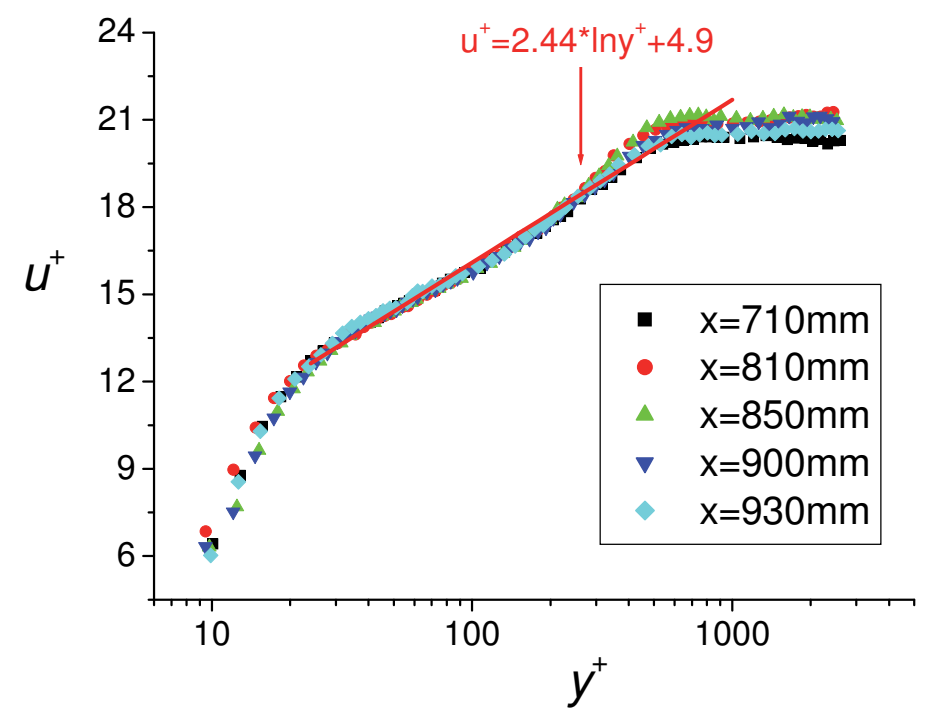

Fig. 7. Mean velocity profile of turbulent boundary layer on a smooth flat plate

\section{Results and discussion}

Various techniques for educing coherent structure component using the information provided by wavelet phase plane have been described in the literature[31][32]. Our object is to partition a turbulence fluctuating signal into two parts using the information provided by wavelet phase plane, one containing coherent structures component and the other containing the residual random component. Two criterions should be assigned for separating the two fluctuating velocity components; one is for the intermittent scale by $F F(a)>3$ and the other for detecting coherent structure by $I(a, t)>L$. The reconstruction can be performed from the wavelet phase plane information detected by these two criterions from the most energetic scale to small scales. Once the dominant scale is determined by the most energetic criterion, the local coherent structure can be identified from the significant maxima amplitudes of wavelet coefficients. The partition then is performed from the most energetic scale to the small-scale reconstruction. Figure 8 presents some single scale coherent structure fluctuating velocity signals reconstructed from each single scale wavelet coefficients. Figure 9 presents the coherent structure velocity signal reconstructed from intermittent wavelet coefficients detected by the intermittency index. 

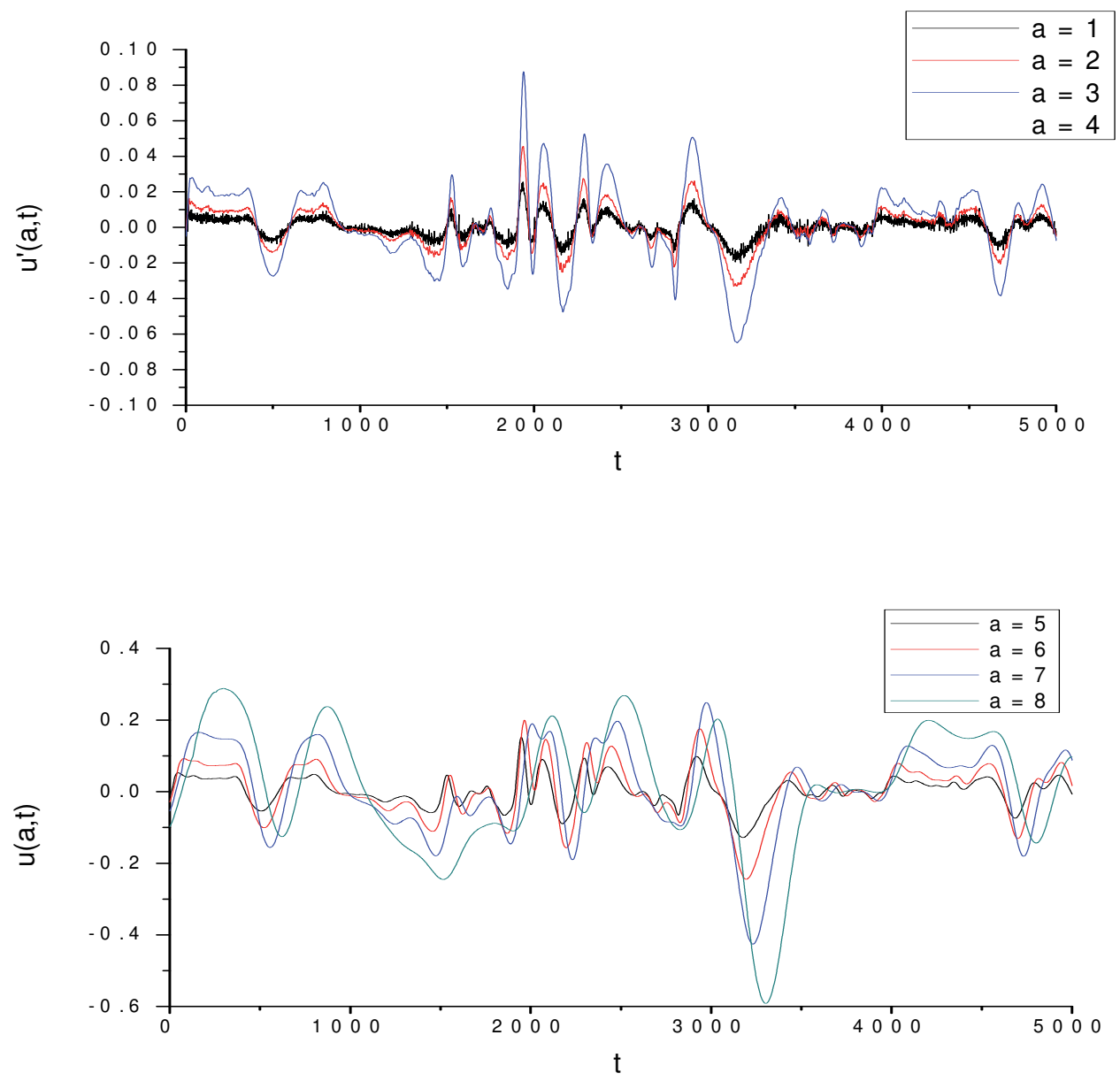

Fig. 8. Time trace of each single scale coherent structure longitudinal fluctuating velocity signal 

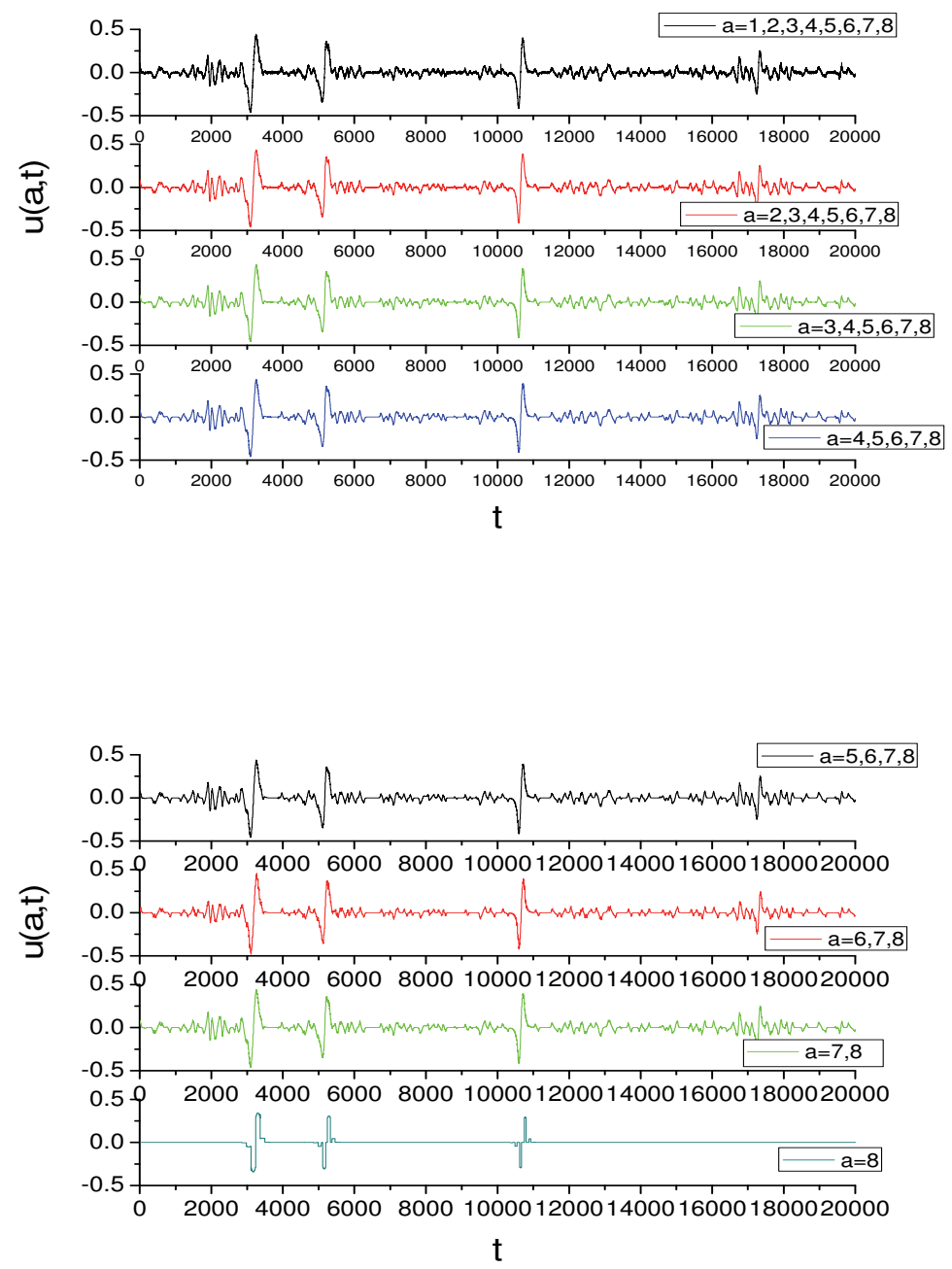

Fig. 9. Time trace of coherent structure velocity signal reconstructed from multi-scale wavelet coefficients 
Figure 10 and 11 shows conditional phase-averaged waveforms of fluctuating velocity component during sweep and eject events for different scales at $\mathrm{y}^{+}=26$ in the buffer region of turbulent boundary layer. The vertical axis in figure 10 and 11 represents the phaseaveraged fluctuating velocity component normalized by the local mean velocity, while abscissa axis represents the time. The shapes of different scales are quite similar though their time scales are different. They are self-organized and self-regenerated. Their development and evolvement process of coherent structures on different scales share some characteristics in common. In figure 10, the downstream (earlier in time) longitudinal fluctuating velocity component of fluid particles is little faster than the upstream (late in time) one, which cause a decelerating or stretching process which means the low-speed streak flow slowly lifts up away from the wall and makes the longitudinal velocity component of the measuring point reduced. In figure 11, the downstream (earlier in time) longitudinal fluctuating velocity component is slow, while the upstream (late in time) longitudinal fluctuating velocity component accelerates, which cause a compressing process, which denotes that high-speed fluid from the outer layer sweeps downwards and makes the local longitudinal fluctuating velocity component of the measured location increased. The time of this process is very short, but the effect is very strong and their behaviors are similar. These universalities provide important clues to understand the mechanism if turbulence production and transport of heat, mass, momentum in wall turbulence.
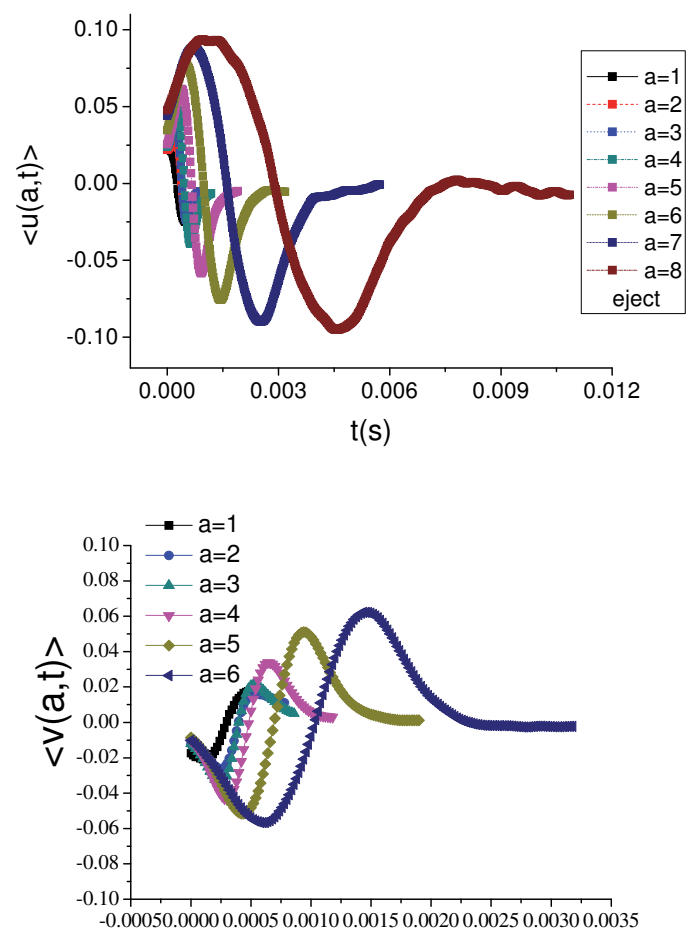

$\mathrm{t}(\mathrm{s})$ 


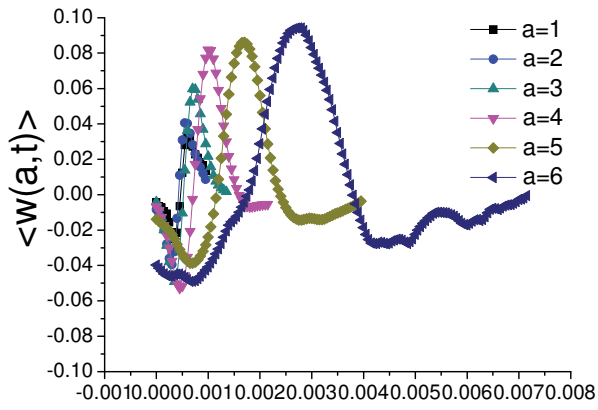

$\mathrm{t}(\mathrm{s})$

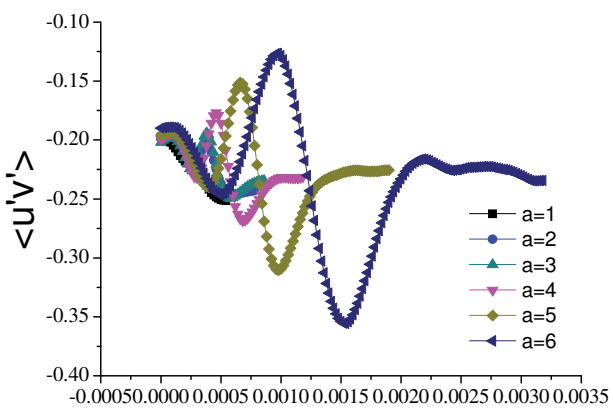

t(s)

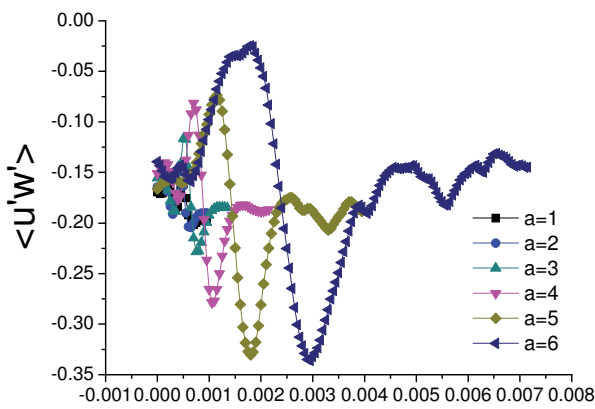

$t(s)$

Fig. 10. Conditional phase-averaged waveform of fluctuating velocity and Reynolds stress for multi-scale coherent structures eject with different scale 

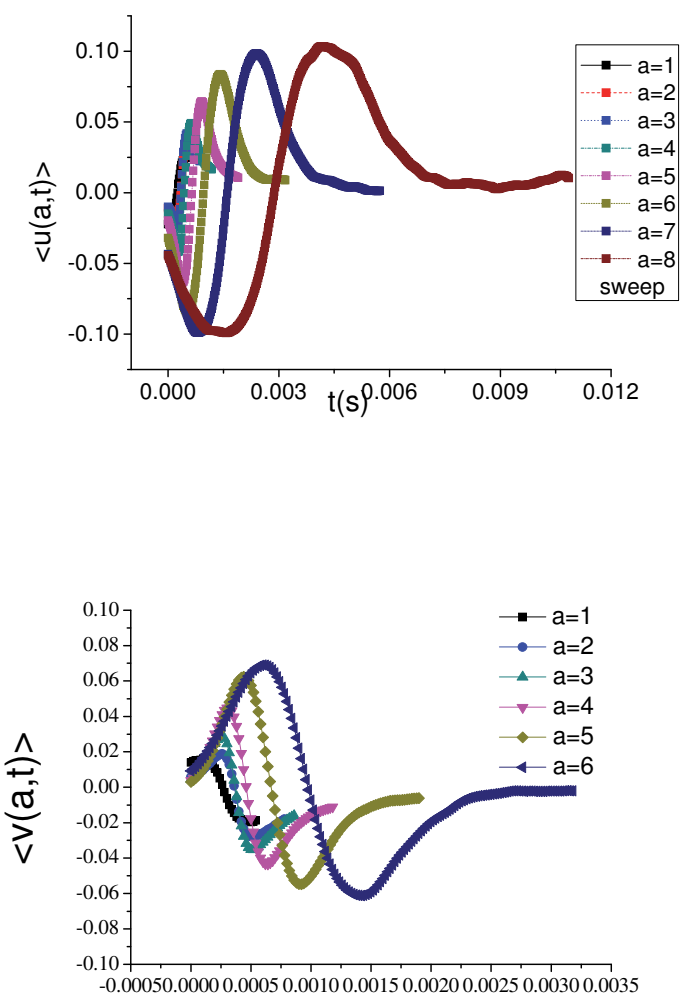

$t(s)$

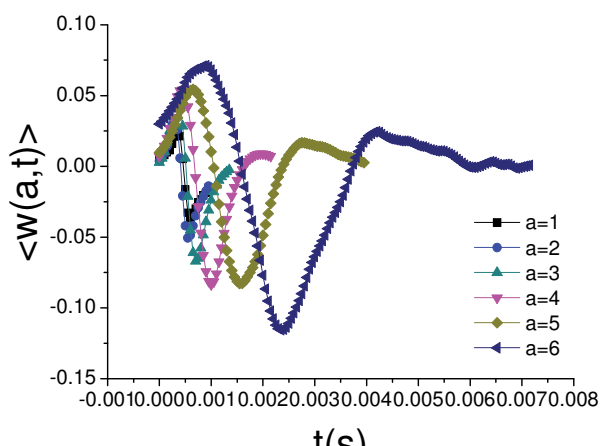

$\mathrm{t}(\mathrm{s})$ 


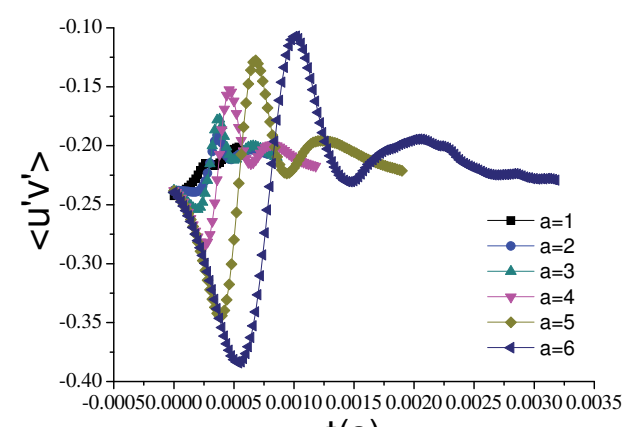

$t(s)$

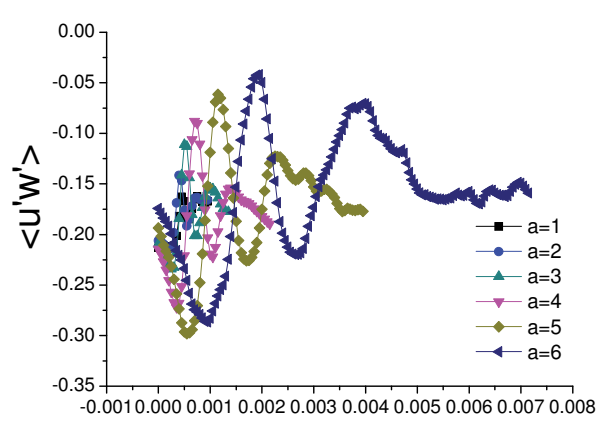

$t(s)$

Fig. 11. Conditional phase-averaged waveform of fluctuating velocity and Reynolds stress for multi-scale coherent structures sweep with different scale

Figure 12 and 13 shows the conditional phase-averaged waveforms of the fluctuating velocity component and Reynolds stress during sweep and eject events for the most energetic scale at different locations across turbulent boundary layer. The shapes of them are similar but their amplitudes are quite different. It indicates that buffer layer is the most important and active region of turbulent boundary layer where coherent structures burst with the largest amplitude. These coherent motions contribute significantly to turbulence production and transport of heat, mass, momentum in the near wall region. The intensities of them decay versus their locations far away from the wall to the out region. Out of the turbulent boundary layer, their intensities are so small and can be neglected. 

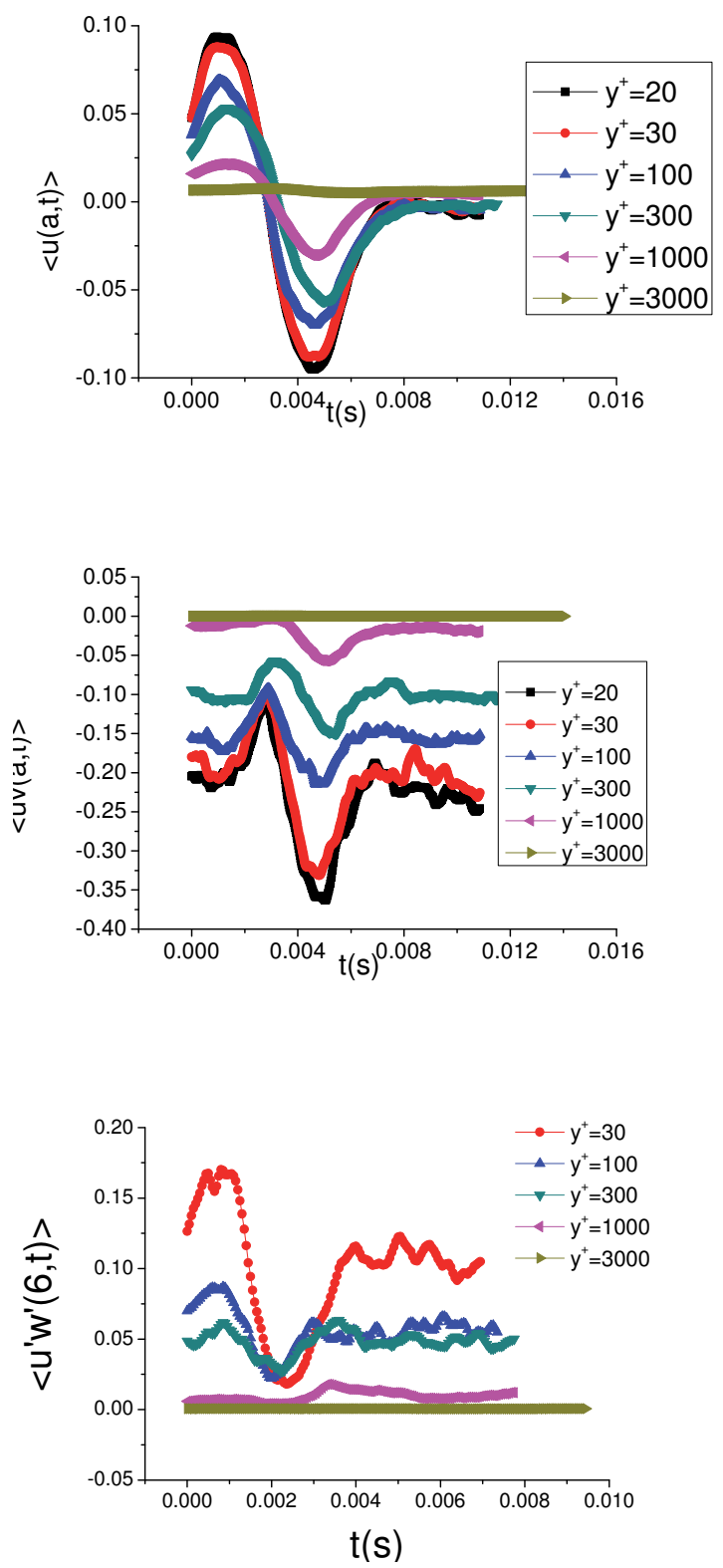

Fig. 12. Conditional phase-averaged waveform of fluctuating velocity and Reynolds stress for multi-scale coherent eddy structures eject for the most energetic scale at different locations in turbulent boundary layer 

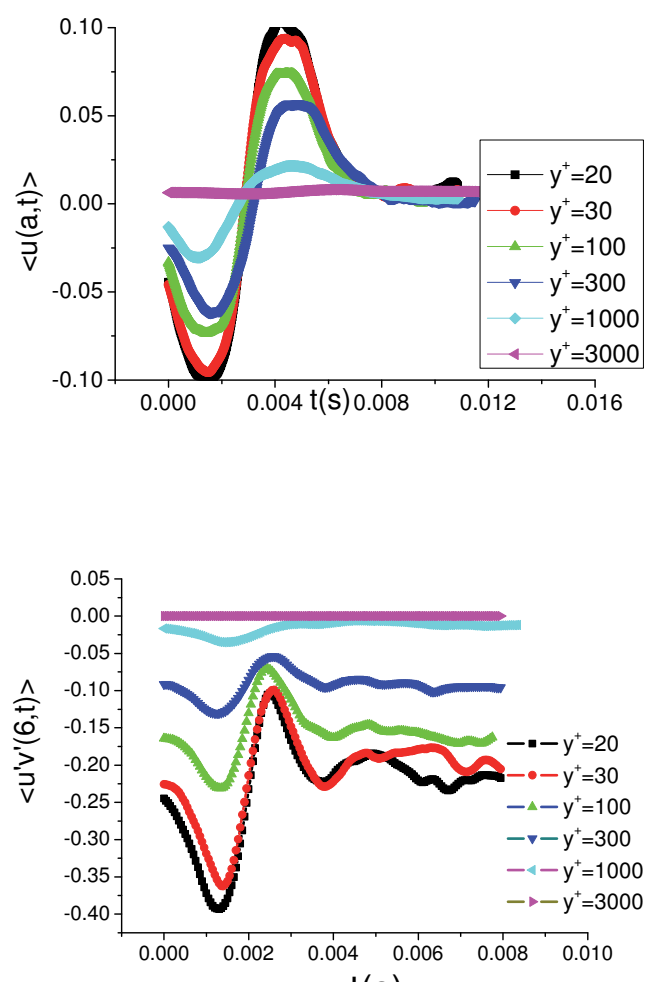

$\mathrm{t}(\mathrm{s})$

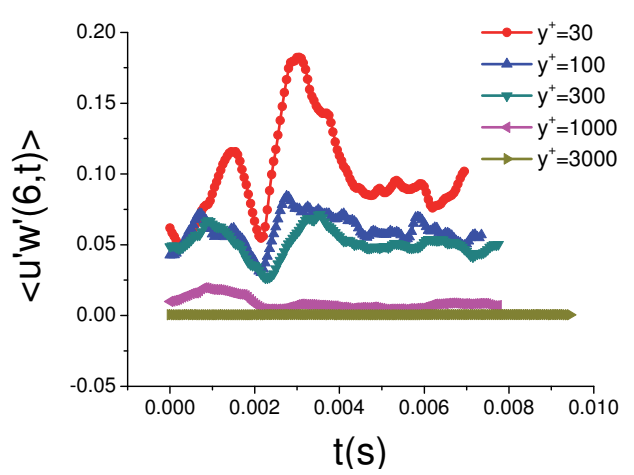

Fig. 13. Conditional phase-averaged waveform of fluctuating velocity and Reynolds stress for multi-scale coherent eddy structures sweep for the most energetic scale at different locations in turbulent boundary layer 
Figure 14 shows the p-th order from the first to the sixth structure functions of wavelet coefficients for $y^{+}=14$ calculated by the extended self-similarity scaling law (ESS)[33][34] within multi-scale coherent structures. The anomalous scaling law calculated by wavelet coefficients is shown in figure 15. Thus the anomalous scaling exponents $\zeta(p, 3)$ can be obtained by linear fit their slopes of these lines. They are substantially different with the Kolmogorov linear scaling lawof $\zeta(p, 3)=p / 3$. Figure 16 shows the $\mathrm{p}$-th order structure functions calculated by ESS after multi-scale coherent eddy structures have been eliminated. It is clear that the scaling exponents without multi-scale coherent eddy structures are exactly fit to the Kolmogorov linear scaling law of $\zeta(p, 3)=p / 3$ in figure 17. This means that multiscale coherent eddy structures are responsible for the intermittence and the anomalous scaling law in turbulence boundary layer. When multi-scale coherent structures are removed by the present conditional sampling technique, the scaling law returns to the linear scaling law $\zeta(p, 3)=p / 3$.

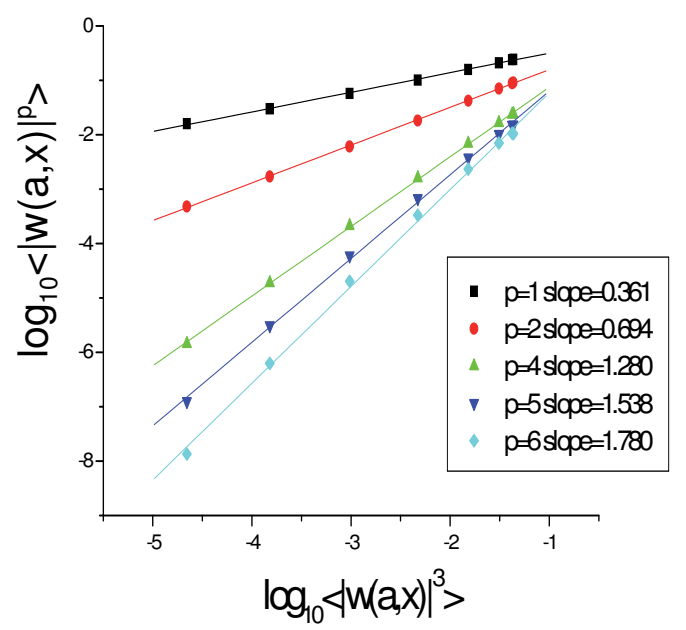

Fig. 14. The p-th order structure functions as a function of the third order structure function calculated using wavelet coefficients within coherent structures at $y^{+}=14$ 


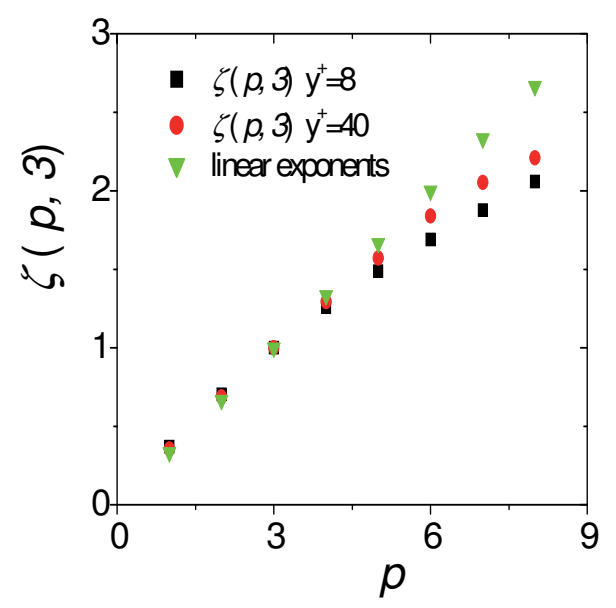

Fig. 15. Relative scaling exponents calculated by wavelet coefficients including coherent structures at $\mathrm{y}^{+=}=8$ and 40 .

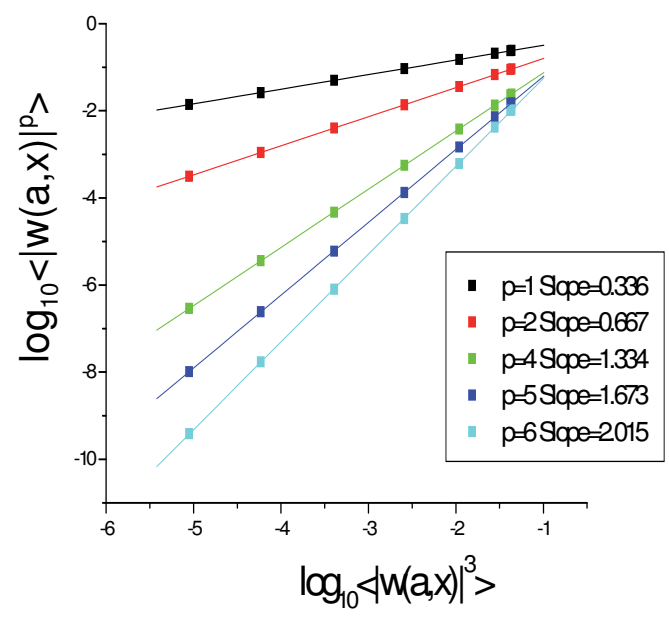

Fig. 16. The p-th order structure functions as a function of the third order structure function calculated using wavelet coefficients without coherent structures 


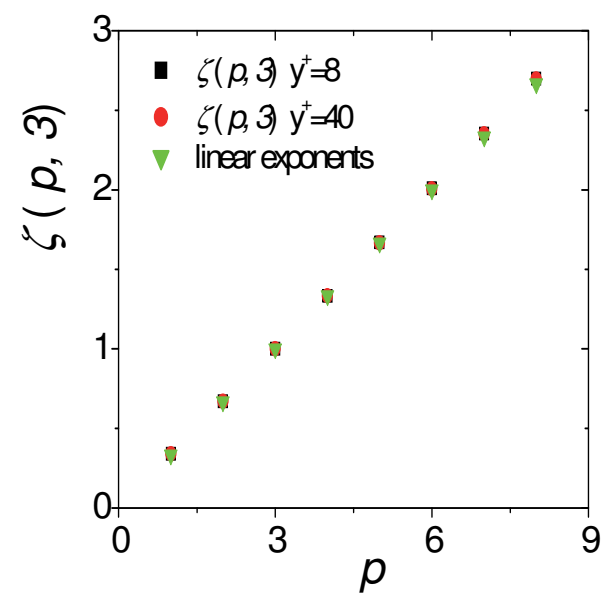

Fig. 17. Relative scaling exponents calculated without coherent structures at $y^{+}=8$ and 40 .

\section{Conclusions}

Turbulent flow is made up of multi-scale eddy structures, and different scale coherent eddy structures exist at different scales that are smaller than the most energetic scale corresponds to the peak of energy contributions. Coherent structures play an important role in turbulence. Coherent eddy structures are much more intensive than non-coherent eddy structures at the same scale.

The energy percentage of large-scale coherent eddy structures at buffer layer is much greater than that of other scales and that of other locations in turbulent boundary layer. Buffer layer is the most important region of turbulent boundary layer for turbulence production with the most active coherent structures.

Besides scales of coherent eddy structures are quite different, phase-averaged evolution shapes of multi-scale coherent eddy structures are similar, which implies the evolvement process of coherent eddy structures on different scales share common characteristics.

Multi-scale coherent eddy structures are responsible for the intermittence and anomalous scaling law in turbulent boundary layer. Multi-scale coherent eddy structures lead to the anomalous scaling law that deviate Kolmogorov linear scaling law. When multi-scale coherent structures are eliminated, the ESS scaling exponents calculated by wavelet coefficients are in agreement well with the Kolmogorov linear scaling law.

\section{Acknowledgment}

Supported by the National Natural Science Foundation of China (Grant No. 100832001 10872145) and science innovation fund of Tianjin University

\section{References}

Kolmogorov A N, Dissipation of energy in the locally isotropic turbulence [J]. Dokl Akad Nauk SSSR, 1941, 32(1): 19 21 
Kolmogorov A N, A refinement of previous hypothesis concerning the local structure of turbulence in a viscous incompressible fluid at high Reynolds number [J]. J Fluid Mech., 1962, 13 (1): 82 85

Jiang Nan, Wang Yu-chun, Shu Wei, Wang Zhen-dong, Experimental study of measurement for dissipation rate scaling exponents in heated wall turbulence[J], Applied Mathematics and Mechanics , 2002, Vol 23, No 9: 1035-1044

Jiang Nan, Wang Zhen-dong, Shu Wei, Extended self-similarity scaling law of multi-scale eddy structure in wall turbulence, Applied Mathematics and Mechanics, 2000 , Vol 21, No 9: 1016-1023

G Ruiz Chavarria, S Ciliberto, C Baudet, E Leveque, Scaling properties of the streamwise component of velocity in a turbulent boundary layer [J]. Physica D, 2000, 141: 183198

F Toschi, G Amati, S Succi, R Benzi, R Piva, Intermittency and structure functions in channel flow turbulence [J]. Phys Rev Lett, 1999, 82(25): 5044 5047

F Toschi, E Leveque, G Ruiz Chavarria, Shear effects in non-homogeneous turbulence [J]. Phys Rev Lett, 2000, 85(7): 1436 1439

Miguel Onorato, Roberto Camussi, Gaetano Iuso, Small scale intermittency and bursting in a turbulent channel flow [J], Physical Review E, Vol. 61, No 2: 1447 1454

She Z-S, Su Wei-dong, Hierarchical structures and scaling in turbulence [J] Advances in Mechanics, 2000, Vol.29, No.3: 289 303 (in Chinese)

Kline S J, Reynolds W C, Schraub F H, Runstadler P W, The structure of turbulent boundary layer [J], J Fluid Mech., 1967, 30: 741-774

Corino E R, Brodkey R S, A visual investigation of the wall region in turbulent flow [J], J Fluid Mech., 1969, 37: 1-30

Kim H T , Kline S J and Reynolds W C. The Production of Turbulence Near a Smooth Wall in a Turbulent Boundary Layer [J] , J. Fluid Mech. , 1971, $50: 133 \sim 160$

Smith C R, Metzler S P, The characteristics of low speed streaks in the near wall region of a turbulent boundary layer [J], J. Fluid Mech. , 1983, 129: 27 54

Liepmann, H. W., Narasimha, R., Turbulence management and relaminarisation, IUTAM symposium, 1987, Bangalore, India

R. Camussi, G. GUJ, Orthonormal wavelet decomposition of turbulent flows: intermittency and coherent structures [J], J. Fluid Mech., Vol. 348: 177 199

Susanta K. Das, Mamoru Tanahashi, Kentaro Shoji, Toshio Miyauchi, Statistical properties of coherent fine eddies in wall-bounded turbulent flows by direct numerical simulation, Theor. Comput. Fluid Dyn. (2006) 20(2): 55“C7

V.A.Sandborn, Measurements of intermittency of turbulent motion in a boundary layer[J], J. Fluid Mech, 1959, 6(2): 221-240

Douady S, Couder Y. \& Brachet M E, Direct observation of intermittency of intense vorticity filaments in turbulence [J]. Phys.Rev.Lett.1991, 67: 983-986

Siggia E D, Numerical study of small-scale intermittency in 3-dimensional turbulence [J], J. Fluid Mech. 1981, 107: 375-406

Vincent A \& Meneguzzi M, The spatial structure and statistical properties of homogeneous turbulence [J]. J. Fluid Mech. 1991, 225: 1-20

She Z-S, Jackson E \& Orszag S A, Intermittent vortex structures in homogeneous isotropic turbulence [J]. Nature 1990, 344: 226-228 
Jiang, N, Chai, Y-B. Experimental investigation of multi-scale eddy structures' intermittency in turbulent flow using probability density function of wavelet coefficients, Journal of Aerospace Power, 20(5), 2005, p 718-724

Marie Farge, Kai Schneider, Coherent vortex simulation (CVS), A semi-deterministic turbulence model using wavelet [J], Flow, Turbulence and Combustion, 2001, Vol.66: 393-426

Charles Meneveau, Analysis of turbulence in the orthonormal wavelet representation [J], J. Fluid Mech., 1991, Vol. 232: 469 520

Farge M., Wavelet transforms and their applications to turbulence [J], Annu. Review Fluid Mech. , 1992, $24: 395-457$

Marie Farge, Nicholas Kevlahan, Valerie Perrier, Eric Goirand, Wavelet and turbulence [J], Proceedings of the IEEE, 1996, Vol.84, No. 4: 639 669

Tennekes H, Lumley J.L. A First Course in Turbulence [M], M.I.T.Press Cambridge, Massachussetts and London, England, 1972

Liu JH, Jiang N, Two phases of coherent structure motions in turbulent boundary layer, Chinese Physics Letters, 2007, Vol 24, No 9: 2617-2620

Liu JH, JIANG N, WANG ZD, et al. Multi-scale Coherent Structures in Turbulent Boundary Layer Detected by Locally Averaged Velocity Structure Functions [J], Applied Mathematics and Mechanics, 2005,Vol 26(4): 456-464

Jiang N, Wang ZD, Shu W. The maximum energy criterion for identifying burst events in wall turbulence using wavelet analysis. Acta Mechanica Sinica, 1997, 29(4): 406 411 (in Chinese)

Jiang N, Liu W, et al. Phase-averaged waveforms of Reynolds stress in wall turbulence during the burst events of coherent structures. Science in China (Series G), 2008, 51(7): $857-866$

Jiang N, Zhang J, Detecting multi-scale coherent eddy structure and intermittency in turbulent boundary layer by wavelet analysis, Chinese Physics Letters, 2005, Vol 22, No 8: 1968-1971

Benzi R,Cilibert S ,Tripiccione R, Extended self-similarity in turbulent flow [J]. Phys.Rev.E, 1993, Vol.48, No.1: R29-R32

Liu W, Jiang N. Three kinds of velocity structure function in turbulent flows. Chinese Physics Letter, 2004, 21(10): 1989-1992 


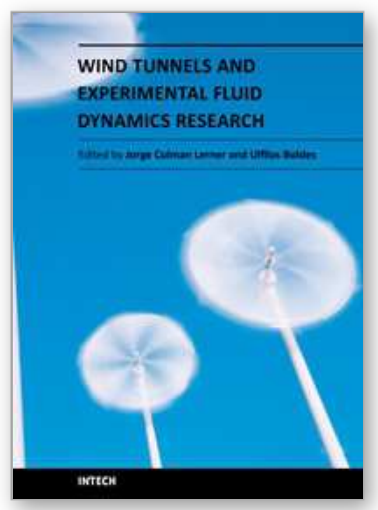

\author{
Wind Tunnels and Experimental Fluid Dynamics Research \\ Edited by Prof. Jorge Colman Lerner
}

ISBN 978-953-307-623-2

Hard cover, 709 pages

Publisher InTech

Published online 27, July, 2011

Published in print edition July, 2011

The book â€œWind Tunnels and Experimental Fluid Dynamics Researchâ€ is comprised of 33 chapters divided in five sections. The first 12 chapters discuss wind tunnel facilities and experiments in incompressible flow, while the next seven chapters deal with building dynamics, flow control and fluid mechanics. Third section of the book is dedicated to chapters discussing aerodynamic field measurements and real full scale analysis (chapters 20-22). Chapters in the last two sections deal with turbulent structure analysis (chapters 23-25) and wind tunnels in compressible flow (chapters 26-33). Contributions from a large number of international experts make this publication a highly valuable resource in wind tunnels and fluid dynamics field of research.

\title{
How to reference
}

In order to correctly reference this scholarly work, feel free to copy and paste the following:

Nan Jiang (2011). Wavelet Analysis to Detect Multi-Scale Coherent Eddy Structures and Intermittency in Turbulent Boundary Layer, Wind Tunnels and Experimental Fluid Dynamics Research, Prof. Jorge Colman Lerner (Ed.), ISBN: 978-953-307-623-2, InTech, Available from: http://www.intechopen.com/books/windtunnels-and-experimental-fluid-dynamics-research/wavelet-analysis-to-detect-multi-scale-coherent-eddystructures-and-intermittency-in-turbulent-bound

\section{INTECH}

open science | open minds

\section{InTech Europe}

University Campus STeP Ri

Slavka Krautzeka 83/A

51000 Rijeka, Croatia

Phone: +385 (51) 770447

Fax: +385 (51) 686166

www.intechopen.com

\section{InTech China}

Unit 405, Office Block, Hotel Equatorial Shanghai

No.65, Yan An Road (West), Shanghai, 200040, China 中国上海市延安西路65号上海国际贵都大饭店办公楼 405 单元

Phone: +86-21-62489820

Fax: +86-21-62489821 
(C) 2011 The Author(s). Licensee IntechOpen. This chapter is distributed under the terms of the Creative Commons Attribution-NonCommercialShareAlike-3.0 License, which permits use, distribution and reproduction for non-commercial purposes, provided the original is properly cited and derivative works building on this content are distributed under the same license. 